\title{
Exotica in CMS
}

\author{
Claudia-Elisabeth Wulz ${ }^{1, a}$, on behalf of the CMS Collaboration \\ ${ }^{1}$ Institute of High Energy Physics of the Austrian Academy of Sciences, \\ Nikolsdorfergasse 18, A-1050 Vienna, Austria
}

\begin{abstract}
Selected results on exotica searches with the CMS detector are presented. The main topics are dark matter, boosted objects, long-lived particles and classic narrow resonance searches. Most of the analyses were performed with data recorded at at centreof-mass energy of $8 \mathrm{TeV}$, but first results obtained at $13 \mathrm{TeV}$ are also shown.
\end{abstract}

\section{Introduction}

At the Large Hadron Collider (LHC) a Higgs boson compatible with the minimal standard model has been discovered. Extensions of this model predict additional Higgs bosons and a wealth of other exotic particles. Searches for new phenomena, however, have been inconclusive so far. The increase of the LHC's centre-of-mass energy from 8 to $13 \mathrm{TeV}$ has opened up new areas of phase space to find or to exclude new particles. In this report we will concentrate on a few captivating recent topics in the field of exotica, including dark matter, boosted objects, long-lived particles and classic narrow resonance searches. Results from proton-proton data recorded with the CMS experiment [1] are presented. An overview of relevant current beyond-standard-model physics results is available on dedicated web pages [2], in particular under "Exotica" and "Beyond 2 Generations".

\section{Dark matter searches}

One of the biggest puzzles today is the nature of dark matter. Beside axions, weakly interacting massive particles (WIMP) are well-motivated candidates. A large range of experiments is currently searching for them in space, underground, or at the surface of the earth. Direct detection is based on the scattering of WIMP dark matter off atomic nuclei, whereas indirect detection makes use of secondary radiation emitted in their pair annihilation. At the LHC one can also search for dark matter particles emerging in collisions of protons. The methods are based on the detection of particles produced in association with the dark matter. They are are complementary to the ones used in direct or indirect searches and are particularly well suited to low-mass WIMPs and spin-dependent couplings. Two scenarios for producing dark matter particles (DM, $\chi$ ) are possible (Fig. 1). The first one assumes a contact interaction, in the framework of an effective field theory. In the second, a mediator, light enough to be produced at the LHC, is exchanged. Since dark matter is known to interact only weakly with standard model particles it can only be detected indirectly, through an imbalance of the total momentum of all particles reconstructed in the plane transverse to the LHC beams. Its magnitude is often

a e-mail: claudia.wulz@cern.ch 

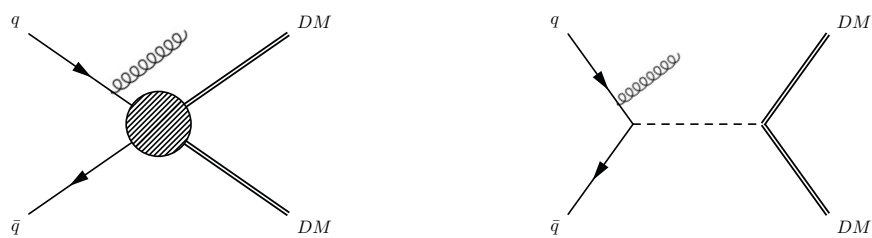

Figure 1. Feynman diagrams for the pair production of dark matter particles for the case of a contact interaction (left) and the exchange of a mediator (right).

denoted as missing transverse energy $\left(E_{\mathrm{T}}{ }^{\text {miss }}\right)$. We focus on the search for single objects (mono-jet, mono-Z, mono-photon) as well as dijets, accompanied by $E_{\mathrm{T}}{ }^{\text {miss }}$.

In the mono-jet search [3] the event selection requires either $E_{\mathrm{T}}{ }^{\text {miss }}>120 \mathrm{GeV}$ calculated from calorimeter information, or a jet reconstructed with the particle-flow technique $[4,5]$ with transverse momentum $p_{\mathrm{T}}>80 \mathrm{GeV}$, and $E_{\mathrm{T}}{ }^{\text {miss }}>105 \mathrm{GeV}$, where $E_{\mathrm{T}}{ }^{\text {miss }}$ is also reconstructed with the particleflow algorithm, but excludes muons. As signal events typically contain jets from initial-state radiation, a second jet is allowed only if its distance in azimuth $(\varphi)$ from the jet with the highest $p_{\mathrm{T}}$ is within 2.5 radians, thus suppressing the dijet QCD background. Events with more than two jets with $p_{\mathrm{T}}>$ $30 \mathrm{GeV}$ and pseudorapidity $|\eta|<4.5$ are discarded, thereby significantly reducing backgrounds from $\mathrm{t} \overline{\mathrm{t}}$ and multijet events. The dominant backgrounds come from $\mathrm{Z}(\rightarrow v v)+$ jets and $\mathrm{W}(\rightarrow \ell v)+$ jets events, and are estimated from data samples of $\mathrm{Z}(\rightarrow \mu \mu)$ and $\mathrm{W}(\rightarrow \mu \nu)$ events, respectively. We studied scalar, vector, axial-vector and tensor interactions for a Dirac DM particle. Fig. 2 (left) shows CMS upper limits for the DM-nucleon cross sections for spin-independent couplings as a function of the mass $\mathrm{M}_{\chi}$, derived from the mono-jet search. Curves from other experiments are overlaid, as well as an area with a possible signal seen by CDMS [6]. Should an effective theory not be valid at LHC energies, we considered the case of an $s$-channel mediator with vector interactions. The resonant enhancement in the production cross section, once the mass of the mediator $(\mathrm{M})$ is within the kinematic range and can be produced on-shell, can be clearly seen in Fig. 2 (centre), which shows results for two dark matter masses and different mediator widths $\Gamma$.
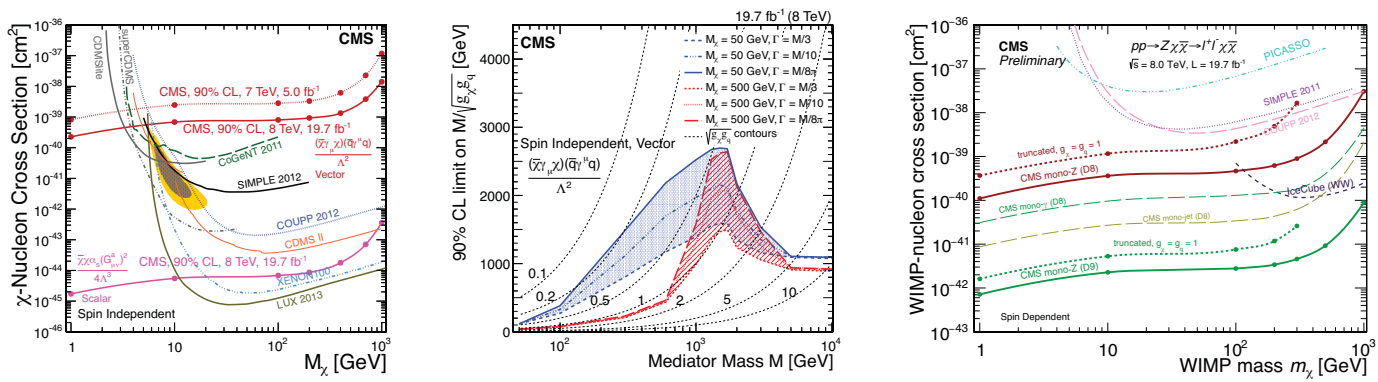

Figure 2. Upper limits on the $\chi$-nucleon cross section, at $90 \%$ confidence level, plotted against the dark matter particle mass and compared with previous results, for spin-independent interactions and the mono-jet channel (left), and for spin-dependent interactions and the mono- $Z$ channel (right); Observed limits on the mediator mass divided by roots of DM and quark couplings, $\mathrm{M} / \sqrt{g_{\chi} g_{q}}$, obtained from the mono-jet analysis (centre). 
Other searches for DM have been performed in the mono-Z [7] and mono-photon [8] channels. Fig. 2 (right) shows CMS limits for spin-dependent interactions obtained in these channels, together with mono-jet results and those of other experiments. D8 and D9 denote specific axial-vector and tensor couplings, respectively.

An innovative DM search using the razor technique in the dijet channel has also been performed, which in particular sheds light on the range of validity of effective field theories [9]. Razor variables, computed from the jet transverse momenta and $E_{\mathrm{T}}{ }^{\text {miss }}$, quantify the balance of the jet momenta. They have been developed in particular to suppress backgrounds from QCD. It has been shown that these variables are also sensitive to direct DM production [10]. The event selection is looser than that used in the mono-jet searches. However, a comparable sensitivity is achieved, as can be seen from Fig. 3, which shows spin-independent (left) and spin-dependent (right) upper limits on the DMnucleon scattering cross section obtained with the dijet razor analysis, together with results from other experiments.
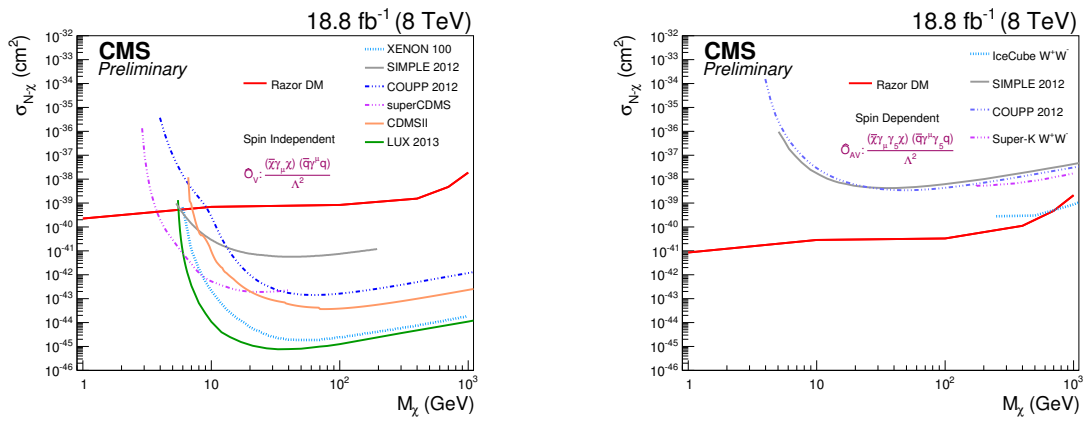

Figure 3. Upper limits at $90 \%$ confidence level on the DM-nucleon scattering cross section as a function of the DM mass in the case of spin-independent vector- (left) and spin-dependent axial-vector currents (right).

As mentioned before, DM production below a cut-off energy scale $\Lambda$ can be described as a contact interaction between two quarks and two DM particles. In the case of $s$-channel production through a heavy mediator, $\Lambda$ is identified with $\mathrm{M} / g_{\mathrm{eff}}$, where $g_{\mathrm{eff}}=\sqrt{g_{\chi} g_{q}}$ is an effective coupling, determined by the couplings of the mediator to quark and DM fields. Lower limits on the cut-off scale are shown in Fig. 4. Following studies presented in Refs. [11-13], we use the variable $R_{\Lambda}$ to define the range of validity of an effective field theory. $\mathrm{R}_{\Lambda}$ was computed as a function of the effective coupling $g_{\text {eff }}$ in the range $0<g_{\text {eff }}<4 \pi$. The contours corresponding to $\mathrm{R}_{\Lambda}=80 \%$ for different values of $g_{\text {eff }}$ are also outlined in Fig. 4. For values of $g_{\text {eff }} \gtrsim 2$, the limits set by the analysis lie above the $R_{\Lambda}=80 \%$ contours.

\section{Long-lived particles}

Long-lived particles are predicted in many beyond-standard-model theories such as in gauge- or anomaly-mediated supersymmetry (SUSY) breaking scenarios, in R-parity violating SUSY and split SUSY models, or in hidden valley scenarios implying a dark sector. Signatures of long-lived particles could be quite unusual. They could imply displaced objects, disappearing or kinked tracks, and delayed objects. Displaced objects would manifest themselves through their origin from a vertex displaced by $\mathrm{O}(10) \mathrm{mm}$ from the primary vertex. Disappearing or kinked tracks would appear after a flight distance of $\mathrm{O}(100) \mathrm{mm}$. Delayed objects refer to very long-lived or stable particles, which 

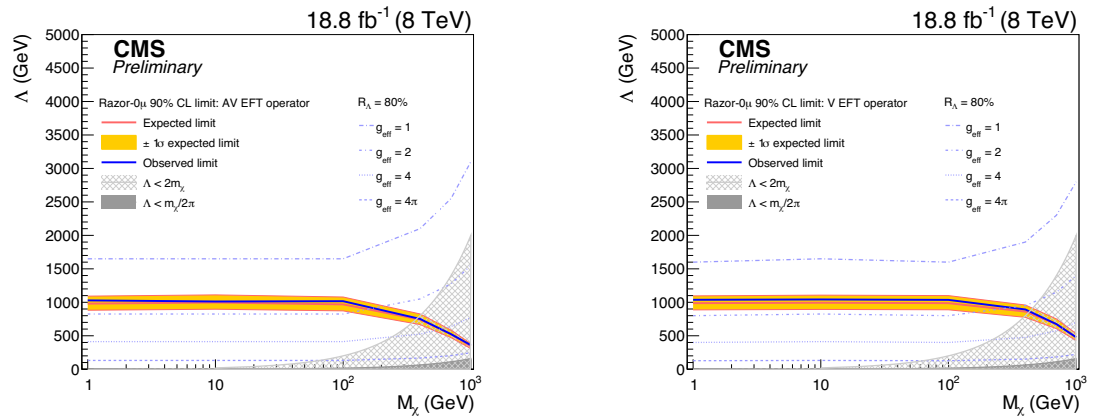

Figure 4. Lower limits at $90 \% \mathrm{CL}$ on the cutoff scale $\Lambda$ as a function of the DM mass in the case of axial-vector (left), and vector currents (right). The validity of the effective field theory for different values of the effective coupling $g_{\text {eff }}$ is quantified by $\mathrm{R}_{\Lambda}=80 \%$ contours.

penetrate the detector and have decay lengths in excess of $\mathrm{O}(1) \mathrm{m}$. Other possible signatures, such as lepton jets, are not discussed in this report. We focus on neutral particles decaying to photons [14] or muons [15], and on a reinterpretation of results on heavy charged particles [16].

In models with gauge-mediated SUSY breaking (GMSB) the long-lived lightest neutralino $\left(\tilde{\chi}_{1}^{0}\right)$ decays to a gravitino $(\tilde{G})$ and a photon $(\gamma)$, represented in Fig. 5 by two possible diagrams of squark and gluino pair-production processes that result in a diphoton final state.
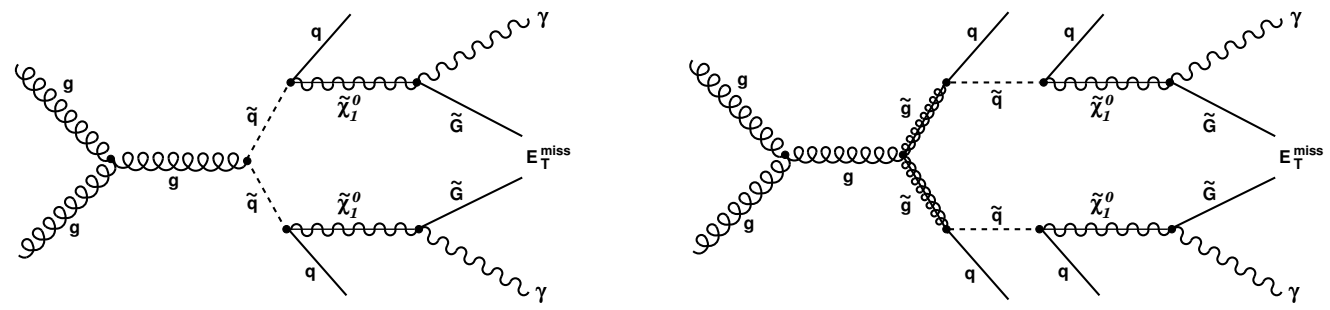

Figure 5. Feynman diagrams for the squark (left) and gluino (right) pair production processes of long-lived lightest neutralinos decaying to gravitinos and photons, resulting in diphoton final states.

The event selection for this decay channel required two photons with at least one converted to an $\mathrm{e}^{+} \mathrm{e}^{-}$pair, two or more jets, and $E_{\mathrm{T}}{ }^{\text {miss }}$. The latter will exhibit a long tail if it originates from gravitons, whereas it will quickly fall off for standard model and instrumental backgrounds. Requiring at least one conversion allows reconstructing the photon direction from the momentum of the electron/positron track pair. By extrapolating the photon direction back to the beam axis the impact parameter of the photon $\left|\mathrm{d}_{\mathrm{xy}}\right|$ can be measured as shown in Fig. 6 (left). A scenario with neutralino decay lengths $\mathrm{c} \tau$ between 0.4 and $100 \mathrm{~cm}$ has been considered. We obtain an exclusion region as a function of both the neutralino mass and its mean lifetime in the context of the SPS8 [17] GMSB scenario, shown in Fig. 6 (right). The mass of the lightest neutralino is restricted to values $\mathrm{m}\left(\tilde{\chi}_{1}^{0}\right)$ $>250 \mathrm{GeV}$, for a mean neutralino decay length of $10 \mathrm{~cm}$. It can also be seen that the mean decay length must be greater than $11 \mathrm{~cm}$, for $\mathrm{m}\left(\tilde{\chi}_{1}^{0}\right)=160 \mathrm{GeV}$. We have obtained roughly a factor of ten improvement with respect to the $7 \mathrm{TeV}$ results [18]. 

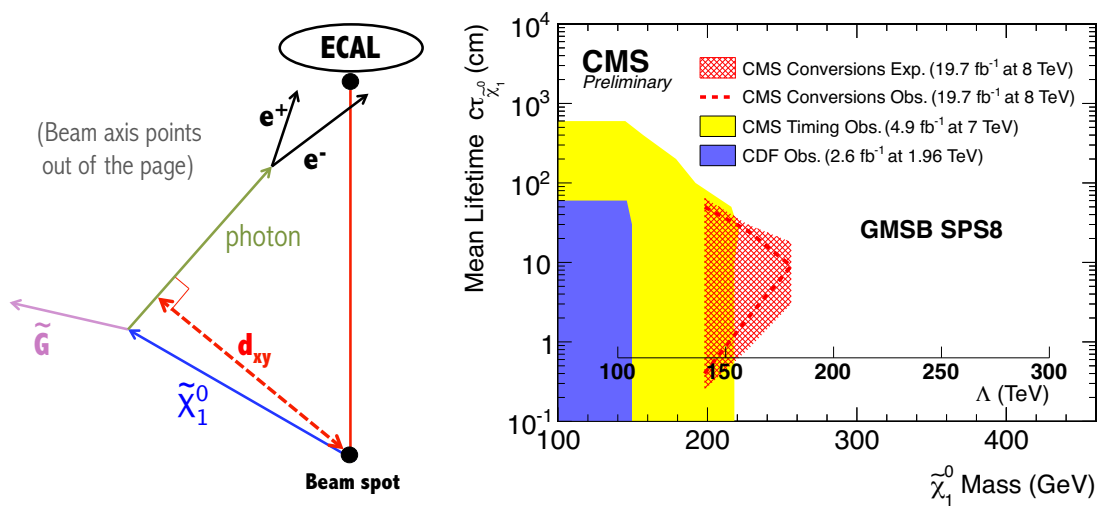

Figure 6. $\tilde{\chi}_{1}^{0}$ decay to $\gamma+\tilde{G}$ in the plane transverse to the LHC beams, with the photon converting to an $\mathrm{e}^{+} \mathrm{e}^{-}$ pair and the subsequent reconstruction of the transverse impact parameter in the electromagnetic calorimeter (left); Exclusion plot in the plane defined by the $\tilde{\chi}_{1}^{0}$ mass and its mean lifetime in the context of the SPS8 GMSB scenario. The scale $\Lambda$ is also shown.

Two models have been considered in a study of long-lived particles decaying to muons [15], with a topology requiring two muons originating from a displaced vertex, detected in the muon chambers only and not in the inner tracker of CMS. The muon reconstruction and selection efficiencies evaluated by Monte Carlo simulations have been cross-checked with cosmics data. Limits have been derived for a non-standard-model Higgs boson $\mathrm{H}$ decaying to two long-lived spin- 0 bosons $\mathrm{X}$ ), and for a squark pair, where each squark decays to a quark and a long-lived neutralino, which in turn decays to two muons and a neutrino in an R-parity-violating scenario. The study is orthogonal to a previous one that used only the tracker [19]. It improves sensitivity to particles that are particularly long-lived. The two analyses have been combined. The example plots of Fig. 7 show limits on the cross sections multiplied by the branching fractions into dimuons for the long-lived particles in the two models, with various combinations of masses for $\mathrm{X}$ bosons and squark/neutralino mass ratios.

A hidden-valley benchmark model has been assumed in a search for displaced jets [20]. Decays of particles from the hidden valley occur via a hidden-sector mediator as they do not couple directly to standard-model particles. A long-lived, spinless, neutral exotic particle $\mathrm{X}^{0}$ which decays to $q \bar{q}$ is assumed. In this model, the $\mathrm{X}^{0}$ is pair-produced in the decay of a non-standard-model Higgs boson, i.e. $\mathrm{H}^{0} \rightarrow 2 \mathrm{X}^{0}, \mathrm{X}^{0} \rightarrow q \bar{q}$, where the Higgs boson is produced through gluon-gluon fusion. This model predicts up to two displaced dijet vertices within the volume of the CMS tracker per event. The topology requirement was therefore two jets originating from the same displaced vertex. An event with this topology is shown in Fig.8 (left). The yellow cones represent the jet pair. The displaced vertex, with a transverse distance from the primary vertex of $5 \mathrm{~cm}$, is defined by the five black tracks belonging to Jet 0 and one black track belonging to Jet 1 . Fig. 8 (right) illustrates expected and observed upper limits for the cross section times branching fraction for $\mathrm{H}^{0} \rightarrow 2 \mathrm{X}^{0}$ with $\mathrm{X}^{0} \rightarrow q \bar{q}$, at $95 \%$ confidence level, for a Higgs boson mass of $1000 \mathrm{GeV}$ and two $\mathrm{X}^{0}$ boson masses, 150 and $350 \mathrm{GeV}$, as a function of decay length.

Examples of so-called heavy stable charged particles (HSCP) are long-lived charginos, sleptons, or R-hadrons, composite colourless states made of a squark or gluino and light quarks or gluons. Search strategies exploit characteristic signatures in the detector, according to the nature of the HSCP. The energy available at the LHC is such that particles with masses greater than $100 \mathrm{GeV}$ and lifetimes 

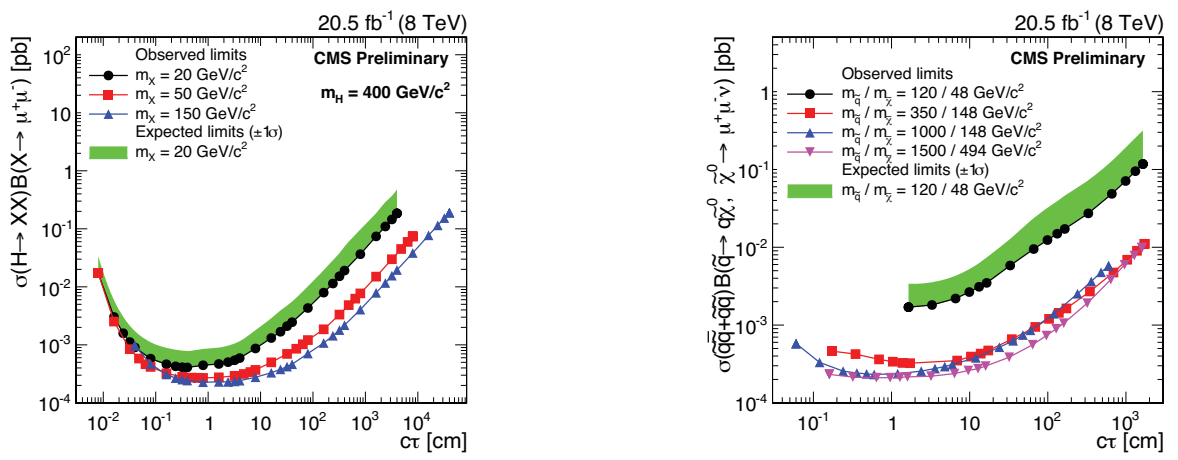

Figure 7. $95 \%$ confidence level upper limits on $\sigma(\mathrm{H} \rightarrow \mathrm{XX}) \mathcal{B}\left(\mathrm{X} \rightarrow \mu^{+} \mu^{-}\right)$for $\mathrm{m}_{\mathrm{H}}=400 \mathrm{GeV}$ with various $\mathrm{X}$ mass points (left). The shaded band shows the $\pm 1 \sigma$ range of variation of the expected limits for the case of a 20 $\mathrm{GeV} \mathrm{X}$ boson mass. Corresponding bands for the other $\mathrm{X}$ boson masses show a similar level of agreement and are omitted for clarity; 95\% CL upper limits on $\sigma(\tilde{q} \overline{\tilde{q}}+\tilde{q} \tilde{q}) \mathcal{B}\left(\tilde{q} \rightarrow q \tilde{\chi}^{0}, \tilde{\chi}^{0} \rightarrow \mu \mu v\right)$ as a function of the neutralino lifetime. Shaded bands show the $\pm 1 \sigma$ range of variation of the expected limits for the case of a $120 \mathrm{GeV}$ squark and a $48 \mathrm{GeV}$ neutralino mass. Corresponding bands for the other squark and neutralino masses show a similar level of agreement and are omitted for clarity.
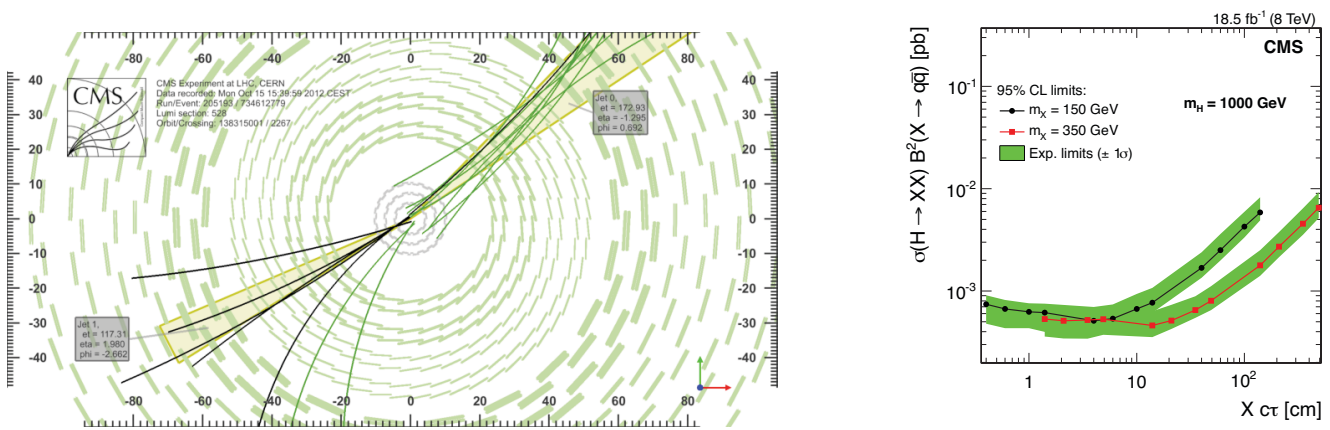

Figure 8. Candidate event with displaced jets (left); Expected and observed upper limits for the cross section times branching fraction for $\mathrm{H}^{0} \rightarrow 2 \mathrm{X}^{0}, \mathrm{X}^{0} \rightarrow q \bar{q}$ (right).

greater than $\mathrm{O}(1)$ ns could be observed as high-momentum tracks with anomalously large energy losses through ionization $(\mathrm{dE} / \mathrm{dx})$. These particles could also be highly penetrating such that the fraction reaching the muon chambers would be sizable. The muon system could therefore be used to help with identification and with the measurement of the long time-of-flight of the particles. Nuclear interactions may also lead to charge exchange, such that the HSCP becomes neutral and can therefore no longer be detected in parts of the tracker or the muon chambers.

We concentrate in this report on a reinterpretation of previous results on long-lived chargino production [21], in the context of the phenomenological minimal supersymmetric standard model (pMSSM) and an anomaly-mediated SUSY breaking model (AMSB) [16]. In the pMSSM, the parameter sub-space for particle masses up to $3 \mathrm{TeV}$ and charginos $\left(\tilde{\chi}^{ \pm}\right)$with a mean proper decay length greater than $50 \mathrm{~cm}$ was considered. Fig. 9 (left) shows the fraction of parameter points excluded as a function of the chargino lifetime. The fraction of excluded model points with a chargino lifetime longer than $1000 \mathrm{~ns}(10 \mathrm{~ns})$ is $100.0 \%$ (95.9\%). Although these values depend on the random point sampling in the 19-dimensional pMSSM parameter space, it is remarkable that a high fraction of the 
points predicting long-lived charginos are excluded. The results represent the first constraints on the pMSSM obtained at the LHC.
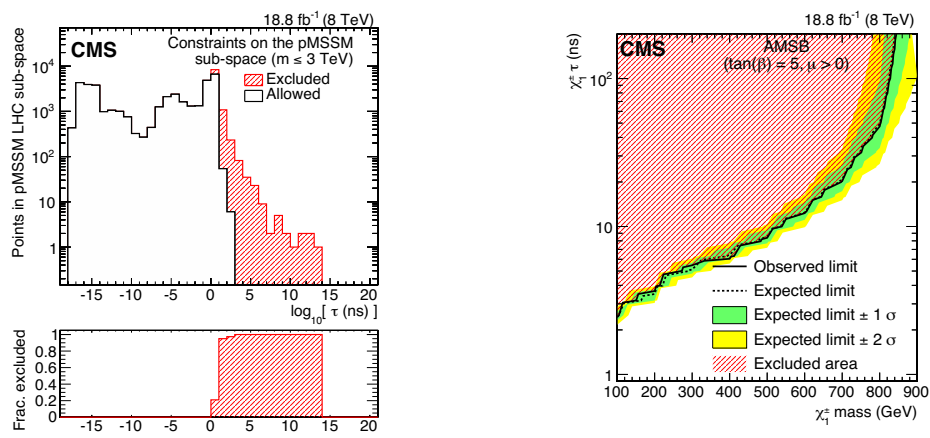

Figure 9. Number of pMSSM parameter points excluded or allowed as a function of the chargino lifetime (left); Observed and expected excluded and allowed regions in the chargino mass and lifetime parameter space in the context of the AMSB model with $\tan \beta=5$ and $\mu>0$ (right).

In the AMSB model, the small mass difference between the lightest chargino $\tilde{\chi}_{1}^{ \pm}$and the lightest neutralino $\tilde{\chi}_{1}^{0}$ can lead to a long chargino lifetime, expected to be of the order of a nanosecond or longer. It is determined by the mass splitting between the two particles. Charginos with lifetimes $\gtrsim 100 \mathrm{~ns}(3 \mathrm{~ns})$ and masses up to about $800 \mathrm{GeV}(100 \mathrm{GeV})$ are excluded at $95 \%$ confidence level, as can be seen from Fig. 9 (right).

\section{Boosted objects}

Particles with a high Lorentz boost have been recognized as tools for discovery already in 1994 [22]. Only recently, however, have the reconstruction techniques become sophisticated enough to exploit them. If a boosted particle disintegrates, the decay products will be emitted close together in space. Jets, in particular, may overlap to a point where they merge into a single "fat" jet. The internal structure of these jets is a key signature to identify boosted objects among the abundant jet production at the LHC. Many searches use a variety of recently proposed substructure observables and techniques such as jet grooming to remove noise and pile-up, or pruning to remove soft, large-angle particles, as well as tagging of $\mathrm{b}$ and $\mathrm{t}$ quarks, or $\mathrm{W}$ and $\mathrm{Z}$ in jets. These new analysis methods led to improvements in sensitivity for high-mass objects up to a factor of about ten.

In the following we describe searches for boosted objects $\mathrm{X}$ decaying to $\mathrm{WH}, \mathrm{ZH}$ or VV, where $\mathrm{V}$ denotes a vector boson $\mathrm{W}$ or $\mathrm{Z}$. We have performed the first search for a resonance decaying to $\mathrm{WH}$, with $\mathrm{W} \rightarrow \ell v$ and $\mathrm{H} \rightarrow \mathrm{b} \bar{b}$ [23]. The search strategy is close to the one for high-mass WW resonances, additional $\mathrm{b}$ tagging requirements. The main backgrounds come from $\mathrm{W}+$ jets, $\mathrm{WW} / \mathrm{ZZ}$ and $\mathrm{t} \overline{\mathrm{t}}$. A narrow resonance $\mathrm{W}^{\prime}$ is assumed. Fig. 10 (left) shows expected and observed upper limits on the product of the $\mathrm{W}^{\prime}$ production cross section and the branching fraction of $\mathrm{W}^{\prime} \rightarrow \mathrm{WH} \rightarrow \mu \nu \mathrm{b} \overline{\mathrm{b}}$ at $95 \%$ confidence level. The cross section for the production of a $W^{\prime}$ in the Little Higgs model [24] and a recently proposed model of a Heavy Vector Triplet [25] HVT, in a specific incarnation called scenario $\mathrm{B}$, multiplied by its branching fraction for the relevant process, is overlaid. The number of events as a function of the reconstructed WH mass for the electron channel and the associated backgrounds are depicted in Fig. 10 (centre). As can also been seen in the right plot of the figure, which shows the $\mathrm{p}$-values for the combined electron/muon channels as a function of the $\mathrm{W}^{\prime}$ mass, a slight but still 

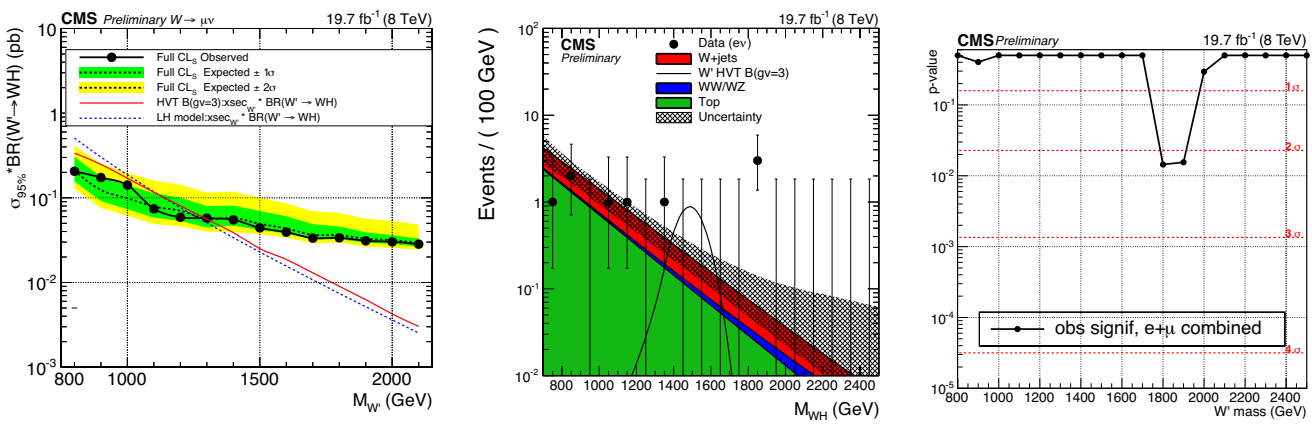

Figure 10. Observed and expected 95\% confidence level upper limits on the product of the $\mathrm{W}^{\prime}$ production cross section and the branching fraction of $\mathrm{W} \rightarrow \mathrm{WH}$ for the muon channel (left); Distributions in $\mathrm{M}_{\mathrm{WH}}$ for data and expected backgrounds for the electron channel (centre); Local significance of the combined electron and muon data probing a narrow $\mathrm{WH}$ resonance (right).

insignificant excess around $1900 \mathrm{GeV}$ is visible. Overall, in the context of the Little Higgs model, we set a lower limit on the $\mathrm{W}^{\prime}$ mass of $1.4 \mathrm{TeV}$. In the HVT model with scenario B we set a lower limit on the $\mathrm{W}^{\prime}$ mass of $1.5 \mathrm{TeV}$ when combining the electron and muon channels.

Another novel analysis using $\tau$ pairs in the boosted regime for the process $\mathrm{X} \rightarrow \mathrm{ZH} \rightarrow \mathrm{qq} \tau \tau$ was performed [26]. Six $\tau$ decay channels were considered, the purely leptonic $\left(\tau_{\mathrm{e}} \tau_{\mathrm{e}}, \tau_{\mathrm{e}} \tau_{\mu}, \tau_{\mu} \tau_{\mu}\right)$, the semileptonic $\left(\tau_{\mathrm{e}} \tau_{\mathrm{h}}, \tau_{\mu} \tau_{\mathrm{h}}\right)$ and the all-hadronic $\left(\tau_{\mathrm{h}} \tau_{\mathrm{h}}\right)$, where the subscripts denote the characterising decay products of the $\tau$. The main backgrounds differ according to the decay categories. For the leptonic ones they are $\mathrm{Z} / \gamma+$ jets, for the semi-leptonic $\mathrm{t} \overline{\mathrm{t}}$ and $\mathrm{W}+$ jets, and for the hadronic one they come from QCD. The on-line event selection required a single jet reconstructed by the anti- $k_{\mathrm{T}}$ algorithm with a distance parameter of 0.5 and $p_{\mathrm{T}}>320 \mathrm{GeV}$, or $H_{\mathrm{T}}>650 \mathrm{GeV}$, where $H_{\mathrm{T}}$ is the scalar sum of the transverse energy of all the jets in the event. Fig. 11 (left) shows the ZH mass distribution together with background estimates in the $\tau_{\mu} \tau_{\mathrm{h}}$ category. A signal for a $\mathrm{Z}^{\prime}$ with mass $\mathrm{M}_{\mathrm{Z}^{\prime}}=1.5 \mathrm{TeV}$ is superimposed. From a combination of all possible decay modes of the $\tau$ leptons, production cross
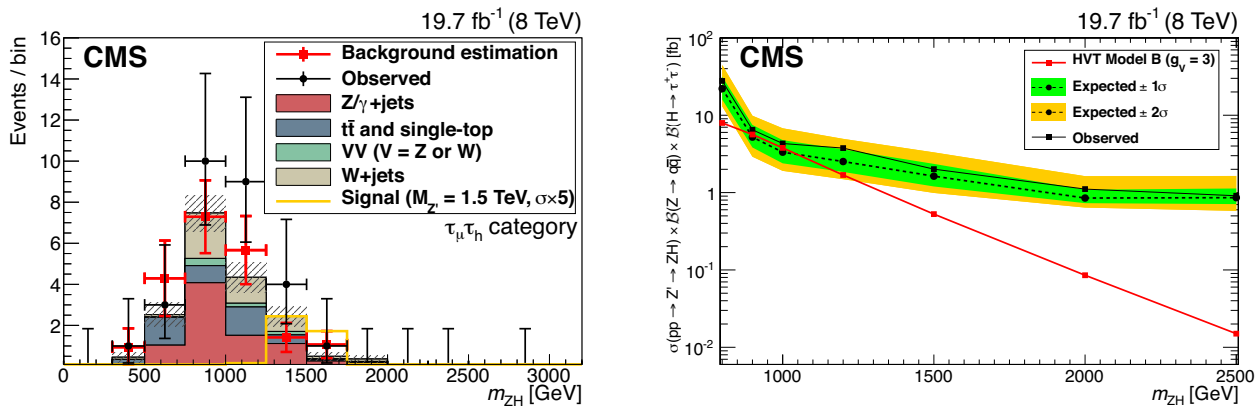

Figure 11. Observed $\mathrm{ZH}$ mass distribution for the $\tau_{\mu} \tau_{h}$ category along with the corresponding Monte Carlo expectations for signal and background, as well as a background estimation derived from data in red (left); Expected and observed upper limits on the $\mathrm{Z}^{\prime}$ cross section times branching fraction for $\mathrm{Z}^{\prime} \rightarrow \mathrm{ZH}$ for the six analysis categories combined (right). 
sections in a range between 0.9 and $27.8 \mathrm{fb}$, depending on the resonance mass in the range 0.8 to $2.5 \mathrm{TeV}$, are excluded at a 95\% confidence level, as can be seen from Fig. 11 (right).

Fig. 12 is a summary plot of several searches for narrow spin-1 resonances decaying to a pair of bosons VV or VH. The curves represent the comparison of the results obtained in different final states and based on data corresponding to an integrated luminosity of $19.7 \mathrm{fb}^{-1}$ recorded in proton-proton collisions at $\sqrt{s}=8 \mathrm{TeV}$. The black dashed curve represents the theoretical prediction for the sum of the production cross sections of the three spin-1 resonances degenerate in mass in the HVT model scenario B. In certain channels there appears to be a slight excess around 1.8 to $1.9 \mathrm{TeV}$ resonance mass.

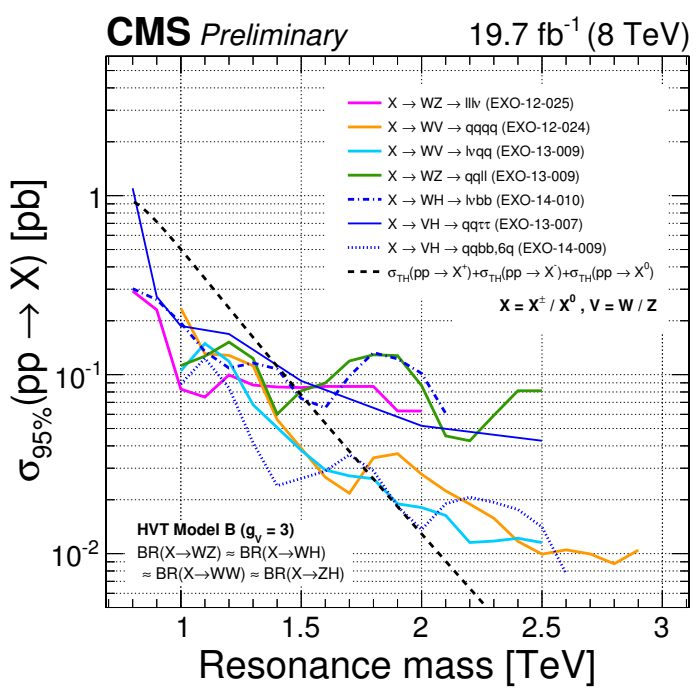

Figure 12. Observed 95\% confidence level upper limits on the production cross sections of neutral $\left(\mathrm{X}^{0}\right)$ and charged $\left(\mathrm{X}^{ \pm}\right)$narrow spin-1 resonances decaying into a pair of bosons VV or VH. The black dashed curve represents the theoretical prediction for the sum of the production cross sections of the three spin-1 resonances degenerate in mass in the HVT model scenario B.

\section{Narrow resonances}

CMS has also performed classic narrow-resonance searches. Interpretations are possible for many exotica scenarios. A first glimpse of $\sqrt{s}=13 \mathrm{TeV}$ data is shown in the dijet spectrum in Fig.13 (left) [27]. The background is estimated from data and parameterized by a smooth function indicated by the red curve. A QCD Monte Carlo prediction, which agrees well with the data, is superimposed in blue. The highest-energy jet pair recorded so far has an invariant mass of $5.4 \mathrm{TeV}$.

With the new data at $\sqrt{s}=13 \mathrm{TeV}$ CMS is already sensitive beyond the $\sqrt{s}=8 \mathrm{TeV}$ reach for resonances with masses larger than about $5 \mathrm{TeV}$, as can be seen from Fig.13 (right), which shows upper limits on cross section times branching fraction times acceptance for dijet resonances of the type gluon-gluon, quark-gluon, and quark-quark, compared to theoretical predictions for string resonances, scalar diquarks, excited quarks, axigluons, colorons, color octet scalars S8, new gauge bosons $\mathrm{W}^{\prime}$ and $\mathrm{Z}^{\prime}$, and Randall-Sundrum gravitons. The mass limit on string resonances at $5.1 \mathrm{TeV}$ in the quark-gluon channel already exceeds the previous limit at $5.0 \mathrm{GeV}$ [28]. 

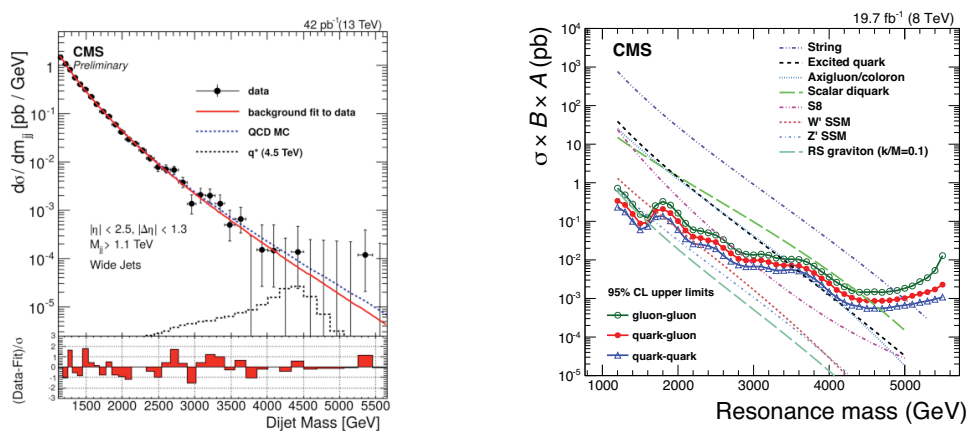

Figure 13. Dijet spectrum for $\sqrt{s}=13 \mathrm{TeV}$ data (left); The observed $95 \%$ confidence level upper limits on $\sigma \times \mathcal{B} \times A$ for dijet resonances of the type gluon-gluon, quark-gluon, and quark-quark, compared to theoretical predictions for several kinds of exotic particles (right).

\section{References}

[1] CMS Collaboration, JINST 3, S08004 (2008)

[2] http://cms-results.web.cern.ch/cms-results/public-results/publications/

[3] CMS Collaboration, EPJC 75, 235 (2015)

[4] CMS Collaboration, CMS-PAS PFT-09-001 (2009)

[5] CMS Collaboration, CMS-PAS PFT-10-001 (2010)

[6] CDMS Collaboration, PRL 111, 251301 (2013)

[7] CMS Collaboration, CMS-PAS EXO-12-054 (2015)

[8] CMS Collaboration, CMS-PAS EXO-12-047 (2014)

[9] CMS Collaboration, CMS-PAS EXO-14-004 (2015)

[10] P. J. Fox, R. Harnik, R. Primulando, and C.-T. Yu, PRD 86, 015010 (2012)

[11] G. Busoni, A. De Simone, E. Morgante, and A. Riotto, PLB 728, 412 (2014)

[12] G. Busoni et al., JCAP 06, 060 (2014)

[13] G. Busoni et al., JCAP 09, 022 (2014)

[14] CMS Collaboration, CMS-PAS EXO-14-017 (2015)

[15] CMS Collaboration, CMS-PAS EXO-14-012 (2015)

[16] CMS Collaboration, EPJC 75, 325 (2015)

[17] C. Allanach et al., EPJC 25, 113 (2002)

[18] CMS Collaboration, JHEP 1211, 172 (2012)

[19] CMS Collaboration,PRD 91, 052012 (2015)

[20] CMS Collaboration, PRD 91, 012007 (2015)

[21] CMS Collaboration,JHEP 07, 122 (2013)

[22] M. Seymour, Z. Phys. C 62,127 (1994)

[23] CMS Collaboration, CMS-PAS EXO-14-010 (2015)

[24] T. Han, H. E. Logan, B. McElrath, and L.-T. Wang, PRD 67, 095004 (2003)

[25] D. Pappadopulo, A. Thamm, R. Torre, and A.Wulzer, JHEP 09, 060 (2014)

[26] CMS Collaboration, PLB 748, 255 (2015)

[27] CMS Collaboration, CMS-PAS EXO-15-001 (2015)

[28] CMS Collaboaration, PRD 91, 052009 (2015) 